\title{
A case of left bundle branch block with chest pain but negative troponin: a prompt for dilated cardiomyopathy?
}

\author{
Authors: Tha Htet $\mathrm{Nyi}^{\mathrm{A}}$ and Hnin $\mathrm{Zaw}^{\mathrm{A}}$
}

\section{Introduction}

Chest pain with left bundle branch block (LBBB) on electrocardiography (ECG) is an alarming presentation in the acute medical unit, even without a rise in troponin. If acute coronary syndrome is unlikely, it is advised to consider common but overlooked differential diagnoses of LBBB such as dilated cardiomyopathy (DCM). The following case, which we came across in the acute medical unit, was a reminder to do so.

\section{Case presentation}

This previously well man in his mid-40s presented with a 1-week history of intermittent central chest pain, which was of a dull ache in nature with no radiation, exacerbated on exertion and by sitting forward. There was no report of preceding prodromal viral illness, no common cold- or flu-like symptoms and no shortness of breath. He had no previous cardiac problems and had never reported chest pain before. He was an ex-smoker and admitted to occasional use of recreational drugs, but no evidence of consistent exposure to cardiotoxic materials. There were no cardiac problems of note in his firstdegree relatives and his exercise tolerance was then essentially normal. On clinical examination, he was relatively hypotensive and bradycardic, but results were otherwise unremarkable. An ECG revealed LBBB with sinus rhythm, no evidence of ischaemia. Troponin was negative two times and blood results were not remarkable, apart from a cholesterol of $5.6 \mathrm{mmol} / \mathrm{L}$. A chest X-ray unveiled a borderline-enlarged heart and subsequent echocardiography revealed a severely dilated left ventricle (LV) with dimensions of $7.6 \mathrm{~cm}$, along with a dilated left atrium and globally severely impaired left ventricular ejection fraction (LVEF) of $20 \%$. For this, he was started on beta-blocker and angiotensin-converting enzyme (ACE) inhibitors. Echocardiography was repeated 1 month later, and showed no improvement in LV systolic function. Meanwhile, it was arranged for him to have a cardiac magnetic resonance imaging (MRI) to find out the underlying cause of DCM, and screening of his first-degree relatives was initiated.

Authors: ${ }^{A}$ Norfolk and Norwich University Hospitals NHS

Foundation Trust, Norwich, UK

\section{Discussion}

A 2016 cohort study illustrated that LBBB commonly presents with dyspnoea (30\%) followed by chest pain $(25 \%) .{ }^{1}$ In addition, a quarter of patients were found to have DCM on echocardiography. ${ }^{1}$ Evidence of LBBB having a negative impact on LVEF in the long term, subsequently leading to DCM, was also reviewed. A retrospective study revealed that almost $10 \%$ (9.8\%) of patients with LBBB with preserved LVEF subsequently developed a significant decline in LV systolic function to an LVEF of approximately $32 \%$ over $4-5$ years. ${ }^{2}$ In terms of management of reduced LVEF in patients with LBBB, cardiac resynchronisation therapy (CRT) plays a more beneficial role ( $35 \%$ of patients in the study sample experienced an improved LVEF $>50 \%$ post-CRT) compared with optimal medical therapy (only $6 \%$ of patients in the study sample benefited from improvement of LVEF after 3 months). ${ }^{3}$

\section{Conclusion}

Whenever ischaemic aetiology is unpromising, it is equally important to consider LBBB-associated non-ischaemic cardiomyopathies, as they can be overlooked and associated with underlying significant LV systolic dysfunction with subtle clinical features. Appropriate and timely specialist input will make a monumental difference to the patient's safety and quality of life, particularly in the long run.

\section{Conflicts of interest}

None declared.

\section{References}

1 Wang NC, Singh M, Adelstein EC et al. New-onset left bundle branch block-associated idiopathic nonischemic cardiomyopathy and left ventricular ejection fraction response to guideline-directed therapies The NEOLITH study. Heart Rhythm 2016;13:933-42.

2 Sharma S, Barot H, Schwartzman A, Patten RD. Incidence of left bundle branch block-associated cardiomyopathy. Circulation 2017;136(Suppl 1):A19033.

3 Bhardwaj R. Etiology and left ventricular functions in left bundle branch block - a prospective observational study. J Assoc Physicians India 2016;64:36-8. 